Acta Phys. Hung. A XX/Y (2006) 1-8

HEAVY ION

PHYSICS

\title{
Hadronization of Expanding QGP
}

\author{
Johann Rafelski ${ }^{1, a}$ and Jean Letessier ${ }^{2, b}$ \\ ${ }^{1}$ Department of Physics, University of Arizona, \\ Tucson, AZ 85718, USA \\ ${ }^{2}$ Laboratoire de Physique Théorique et Hautes Energies \\ Université Paris 7, 2 place Jussieu, 75251 Cedex 05, France \\ Received 4 November 2005
}

\begin{abstract}
We discuss how the dynamics of an exploding hot fireball of quarkgluon matter impacts the actual phase transition conditions between the deconfined and confined state of matter. We survey the chemical conditions prevailing at hadronization.
\end{abstract}

Keywords: QGP, strangeness, supercooling, hadronization

PACS: 12.38. Mh,24.10. Pa,25.75.-q

\section{Introduction}

It is an open question if, within the short time available in a laboratory heavy ion collision experiment, $10^{-22}-10^{-23} \mathrm{~s}$, the confined color frozen nuclear phase can melt and turn into the deconfined quark-gluon plasma (QGP) state of matter. Assuming this is the case, we address here the question how the dynamical evolution influences the conditions present at break-up of the deconfined phase.

We will argue that strangeness production within a fireball of rapidly expanding matter can strengthen the phase transition between QGP and hadron gas (HG). This is of importance since the QCD thermal state turns out to remain below a first order transition for a physical set of masses of two low mass $m_{q} / T \rightarrow 0$, and one semi-heavy quark of mass $m_{s} / T \simeq 1$. This is illustrated, on left, in Fig. 10 [1].

The presence of a positive quark (baryo-)chemical potential $\mu_{q}$ increases the pressure, and the effect is important when $T \simeq \mu_{q}$. The nearly massless quarks respond more strongly to the finite baryo-chemical potential, than do massive hadrons, and that is why the quark-phase response matters more. For this reason, a 1st-order phase transition is expected to arise at a finite value of the chemical potential, as is seen on right in Fig. 10[2, 3, 4]. Baryon density, as expressed by the value of the baryo-chemical potential $\mu_{\mathrm{B}}=3 \mu_{q}$, is relatively low at RHIC, where $\mu_{\mathrm{B}} \simeq 25 \mathrm{MeV}$. 

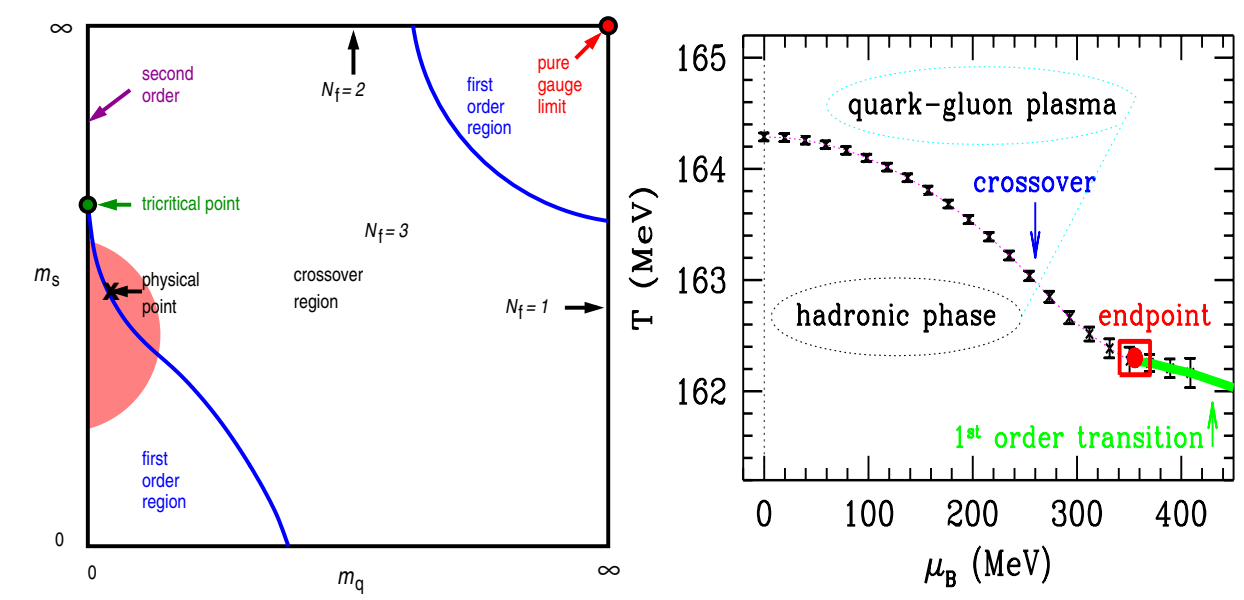

Fig. 1. QCD thermal phase boundaries, left in the $m_{q}-m_{s}$ plane [1], and right in the $T-\mu_{\mathrm{B}}$ plane, for $2+1$ flavors [ 2 .

At the much higher energy at LHC, the expected value of baryo-chemical potential is of magnitude $\mu_{\mathrm{B}} \simeq 1-3 \mathrm{MeV}$ [ [5].

We recognize, in Fig. 1 that if we could compensate even partially the effect of the finite strange quark mass, this would suffice to move the physical point to small and even vanishing baryo-chemical potential. In chemical non-equilibrium, the Fermi distribution function takes the form [ []

$$
\frac{d^{6} N_{s}}{d^{3} p d^{3} x}=\frac{\gamma_{s} e^{-\sqrt{m_{s}^{2}+p^{2}} / T}}{1+\gamma_{s} e^{-\sqrt{m_{s}^{2}+p^{2}} / T}} .
$$

For $\gamma_{s}>1$, there is compensation of the finite mass effect [ [7]. In the limit $\gamma_{s} \rightarrow$ $e^{m_{s} / T}$, the strange quark would play a similar role as a light quark and we expect a rather strong first order phase transition even at vanishing baryo-chemical potential.

The question we address first is, if such an over-saturation of the strangeness phase space near to the phase transition is possible in the dynamical environment we study. We also look at any further dynamical effects associated with the explosive flow of deconfined matter, and search to understand if this assists development of the singular phase behavior. We close with a discussion of constraints imposed by approximate conservation of strangeness and entropy in hadronization.

\section{QGP Phase Strangeness Over-saturation}

The fireball of QGP, created in heavy ion collisions, is initially significantly more dense and hot than after its expansion towards final breakup condition. This expansion dilutes the high strangeness yield attained in the initially very dense and hot 
phase. Contrary to intuition, this can result in an over-saturation of the chemical abundance, even if the initial state is practically strangeness free.

Considering the yield of strange quark pairs in Boltzmann approximation, at a temperature $T=T_{e}$, time $t=t_{e}$, within the volume $V=V_{e}$, we have:

$$
N_{s}\left(t_{e}\right)=\gamma_{e} \frac{2 V_{e} T_{e}^{3}}{\pi^{2}} x_{e}^{2} K_{2}\left(x_{e}\right), x_{e}=\frac{m_{s}\left(T_{e}\right)}{T_{e}} .
$$

We have implied above that the mass of strange quark is $T$-dependent as the scale of energy at which its value is determined is in the domain where a rapid mass change occurs [ 8 ]. We choose the value of $T_{e}$ to be the point where the system has nearly reached chemical equilibrium abundance in QGP, with $\gamma_{e}^{\mathrm{QGP}}=1$ (superscript QGP reminds us that we are considering the deconfined phase). We assume that the continued expansion preserves entropy as is appropriate for an ideal liquid. Since the entropy is governed by essentially massless quark-gluon quanta, this implies that $V T^{3}=$ Const. . Model calculations show that, for typical values of $T_{e}$, the change in the absolute number of strange quark pairs has essentially stopped for $T<T_{e}$. Then, for $t_{1}>t_{e}$ :

$$
\frac{N_{s}\left(t=t_{1}\right)}{N_{s}\left(t=t_{e}\right)} \simeq 1=\gamma_{s}\left(T_{1}\right) \frac{x_{1}^{2} K_{2}\left(x_{1}\right)}{x_{e}^{2} K_{2}\left(x_{e}\right)} .
$$

Since the function $x^{2} K_{2}(x)$ is a monotonically falling function (see Fig. 10.1, p197, in Ref. [ [8]), in general $\gamma_{s}^{\mathrm{QGP}}\left(t_{1}\right)>1$. In the non-relativistic limit $m>T$, that is $x>1$ :

$$
\gamma_{s}^{\mathrm{QGP}}\left(T_{1}\right)=\frac{x_{0}^{2} K_{2}\left(x_{0}\right)}{x_{1}^{2} K_{2}\left(x_{1}\right)}>1 .
$$

How large can $\gamma_{s}^{\mathrm{QGP}}\left(T_{1}\right)$ be? Should the QGP breakup occur from a supercooled state with $T_{1}=140 \mathrm{MeV}$, then it is appropriate to consider $m_{s}\left(T_{1}\right) / m_{s}\left(T_{0}\right) \simeq 1.5^{-}$ 2 , with $T_{0} \simeq 210 \mathrm{MeV}$. To convert from temperature dependence to the energy scale dependence, we set $\mu \simeq 2 \pi T$ and thus, for $m\left(T=T_{0}\right)$, we need an estimate of $m_{s}(\mu=1.3 \mathrm{GeV})$. The PDG value is $m_{s}(\mu=2 \mathrm{GeV})=80-130 \mathrm{MeV}$. Thus, assuming $m_{s}\left(T_{0}\right) \simeq 140$, we find $\gamma_{s}^{\mathrm{QGP}}\left(T_{1}\right)>1.5$. As this example shows, it is possible to nearly compensate the effect of the strange quark mass, see Eq. (11).

We studied quantitatively the possibility of strangeness (over)population at RHIC, prior to the first experimental results becoming available [ 9]. We found $\gamma_{s}^{\mathrm{QGP}}\left(T_{1}\right)<1.2$. We believe that even greater values of $\gamma_{s}^{\mathrm{QGP}}$ can be arrived at: we considered the instantaneous establishment of transverse expansion speed, which cut into the lifespan of the QGP phase and thus reduced the production yield of strangeness. In addition, the quark mass chosen was significantly above the currently preferred value $\left(m_{s}=105 \pm 25 \mathrm{MeV}\right)$ which has further cut into the rate of production of strangeness. However, the lower value of $m_{s}$ also couples strangeness more strongly to the expansion dynamics which could help keep it more in chemical equilibrium. 
There are studies which found considerably smaller values of $\gamma_{s}^{\text {QGP }}$ at RHIC [ 10. 11, 12. In part, this is due to employment of dynamical QGP equilibration models that do not allow gluon chemical equilibration, a prerequisite for abundant strangeness formation. Rapid gluon equilibration processes are presently not fully understood. We assumed rather rapid glue chemical equilibration with relaxation time shorter than $1.5 \mathrm{fm}$. We further note somewhat unrealistically low values used for the coupling constant $\alpha_{s}$ - we use QCD measured value: $\alpha_{s}\left(M_{Z}\right)=0.118$ and use two and higher loop evolution to obtain the low energy scale values [13. We believe that if not at RHIC, then at LHC, we should expect at QGP hadronization a substantial phase space excess of strange quarks.

Though $\gamma_{s}$ is a useful tool to understand how strangeness can help to facilitate phase transition, it is important to remember that it is merely a quantity which relates actual abundance of strangeness to the equilibrium abundance,

$$
n_{s} \simeq \gamma_{s} n_{s}^{\infty}, \quad n_{s}^{\infty} \equiv n_{s}\left(\gamma_{s}=1\right) .
$$

A more direct way to see how $2+1$ flavors turns into a 3 -flavor QCD is to note that we hope and expect that the dynamics of fireball expansion could help us to reach the condition,

$$
n_{s}\left(T_{1}\right) \simeq n_{u}\left(T_{1}\right) \simeq n_{d}\left(T_{1}\right),
$$

even if at point of chemical equilibrium, $n_{s}\left(T_{e}\right) \simeq 0.5 n_{i}\left(T_{e}\right), i=u, d$.

In physical terms, relative importance of strangeness increases since strangeness yield is not reduced along with light quark and gluon yields during the dense matter expansion. Consider the buildup of collective expansion of the fireball of matter which requires conversion of thermal energy into kinetic energy of collective motion. The entropy density decreases, but the expansion assures that the total entropy remains constant, or slightly increases. As the energy is transfered into the transverse expansion, strange quark pair yield, being weaker coupled, remain least influenced by this loss which mainly consumes light quarks and gluons.

We thus conclude that in the event the initial conditions present in QGP are sufficiently extreme to generate strangeness rapidly and abundantly, one can expect over-population of strangeness in the final QGP breakup. This condition can facilitate the occurrence of a phase transition at small and vanishing baryon density.

\section{Explosive Matter Flow}

The covariant characterization of the Gibbs condition for the force balance between phases $A$ and $B, P \equiv P_{A}-P_{B}=0$ requires in the relativistic dynamics the introduction of the energy-momentum tensor $T^{\mu \nu}$. In the laboratory rest frame its components are:

$$
\widehat{T}^{i j}=P \delta_{i j}, \widehat{T}^{i 0}=\widehat{T}^{0 i}=0, \widehat{T}^{00}=\varepsilon .
$$

where $\varepsilon$ is the energy density. The latin indices as usual refer to space component $i=1,2,3$ and the wide hat indicates the laboratory frame. 
Gibbs considered a space-like surface along which pressure difference had to vanish. This surface is invariantly characterized by a normal four-vector $n^{\mu}=(0, \vec{n})$. We take as the covariant, frame of reference independent statement of the Gibbs condition:

$$
T^{\mu \nu} n_{\mu} n_{\nu} \equiv T_{A}^{\mu \nu} n_{\mu} n_{\nu}-T_{B}^{\mu \nu} n_{\mu} n_{\nu}=0 .
$$

We now consider matter subject to expansion flow. $\vec{v}$ is the velocity of the local matter element, and its 4 -velocity is $u^{\mu}=(\gamma, \vec{v} \gamma), \quad \gamma=1 / \sqrt{1-v^{2}}$. The natural presence of two Lorentz vectors $n^{\mu}, u^{\mu}$, assures that we cannot transform away the effect of motion, the colored state pushes against Gibbs surface where the phase boundary is located. We recognize this as the hadronization hyper-surface, where the final state hadrons are born.

The components of interest in the energy momentum tensors are the pressure components:

$$
T^{i j}=P \delta_{i j}+(P+\varepsilon) \frac{v_{i} v_{j}}{1-\vec{v}^{2}} .
$$

The balance of forces between the deconfined QGP and confined hadron phase comprises the effect of the vacuum which confines color. This pressure, introduced in bag models of hadrons for the first time, is traditionally referred to as the bag constant, $\mathcal{B} \simeq(0.2 \mathrm{GeV})^{4}$ [14]. This vacuum structure can be represented within $T^{i j}$ by: $P_{V}=-\mathcal{B}, \quad P_{V}+\varepsilon_{V}=0$. The vacuum structure component is thus not entering the dynamical flow term, the last term in Eq. (9).

We obtain from Eq. [8]:

$$
\mathcal{B}=P_{\mathrm{p}}+\left(P_{\mathrm{p}}+\varepsilon_{\mathrm{p}}\right) \frac{\kappa v^{2}}{1-v^{2}}, \quad \kappa=\frac{(\vec{v} \cdot \vec{n})^{2}}{v^{2}},
$$

where, $P_{\mathrm{p}} \equiv P_{\mathrm{p}}^{\mathrm{QGP}}-P_{\mathrm{p}}^{\mathrm{HG}}, \varepsilon_{\mathrm{p}}^{\mathrm{p}} \equiv \varepsilon_{\mathrm{p}}^{\mathrm{QGP}}-\varepsilon_{\mathrm{p}}^{\mathrm{HG}}$, are the particle pressure and energy density components in the pressure and energy density, from which any vacuum term has been separated. For $\vec{v} \rightarrow 0$, the conventional Gibbs condition reemerges, $P_{\mathrm{p}}^{\mathrm{HG}}=P_{\mathrm{p}}^{\mathrm{QGP}}-\mathcal{B}$.

Eq. (10) describes the pressure of motion of color charged matter against the vacuum structure which is pushed out as color cannot exist there [15]; we can speak of color wind [16]. The magnitude of the flow effect on the value of temperature of the phase boundary is relatively large. This is due to a rather large, in comparison to the pressure, energy density. In presence of a phase transition, we can expect that the discontinuity in particle energy is about $6-7$ times greater than the discontinuity in the particle pressure in Eq. (10) - we recall that only the total pressure is continuous as expressed by the covariant Gibbs condition, Eq. (8).

As a consequence, we expect a significant down-shift in the critical temperature: the moving colored fields cause the quark matter to super-cool by as much as $\Delta T=$ $25 \mathrm{MeV}$ [15]. This means that if the equilibrium phase crossover were to occur at $T=165 \mathrm{MeV}$, the dynamical cross over would be postponed to the value $T=140$ $\mathrm{MeV}$. We do not know at this time if the collective and rapid outflow of QCD can turn a cross-over into a phase-transition. 


\section{Strangeness and Entropy}

Most of entropy of the deconfined state arises in the initial parton reactions and rapidly following thermalization. Entropy is enhanced by color bond breaking, and presence of gluons [ 17, 6], it is a probe of the early evolution of the QGP phase. Subsequently, gluon fusion reactions form strangeness [18]. Both strangeness and entropy are nearly conserved in the evolution towards hadronization and thus the final state hadronic yield analysis value for $s / S$ is closely related to the thermal processes in the fireball at $\tau \simeq 1-2 \mathrm{fm} / \mathrm{c}$. We believe that, for reactions in which the system approaches strangeness equilibrium in the QGP phase, one can expect a prescribed ratio of strangeness per entropy, the value is basically the ratio of the QGP degrees of freedom.

We estimate the magnitude of $s / S$ deep in the QGP phase, considering the hot stage of the reaction [19]. For an equilibrated non-interacting QGP phase with perturbative properties:

$$
\frac{s}{S}=\frac{\left(3 / \pi^{2}\right) T^{3}\left(m_{s} / T\right)^{2} K_{2}\left(m_{s} / T\right)}{\left(32 \pi^{2} / 45\right) T^{3}+n_{\mathrm{f}}\left[\left(7 \pi^{2} / 15\right) T^{3}+\mu_{q}^{2} T\right]} \rightarrow 0.027 \frac{f\left(\alpha_{s}\right) \gamma_{s}}{0.38 \gamma_{\mathrm{G}}+0.12 \gamma_{s}+0.5 \gamma_{q}},
$$

where to obtain the last expression we used for the number of flavors $n_{\mathrm{f}}=2.5$, and for insignificant baryon density, we neglected the $\mu_{q}$ term in denominator. The numerical value of the coefficient follows for $m_{s} / T=1$. All $\gamma_{i}$ refer, here, to QGP phase. $f\left(\alpha_{s}\right)$ is an unknown factor that accounts for the interaction effects - these maybe canceling, with $f\left(\alpha_{s}\right) \rightarrow 1$. Seen the dependence of Eq. (11) on $\gamma_{i}$, we expect to see a gradual increase in $s / S$ as the QGP source of particles approaches chemical equilibrium with increasing collision energy and/or increasing number of participating nucleons.

Since the ratio $s / S$ is established early on in the reaction, the above relations, and the associated chemical conditions we considered, probe the early hot phase of the fireball. How does this simple prediction compare to experiment? An analysis of experimental data [ 17 19, both as function of energy and participant number shows a gradual increase towards the value predicted by Eq. (11), and thus, one is tempted to conclude that strangeness nearly saturates at RHIC top energy for most central collisions.

We can consider a more direct observable which traces out qualitatively $s / S$ : the yield of $\mathrm{K}^{+}$closely follows that of strangeness $s=\bar{s}$, and the yield of $\pi^{+}$is related to the total multiplicity $h$, and thus entropy $S$, the experimental observable of interest is the ratio $\mathrm{K}^{+} / \pi^{+} \propto \bar{s} / \bar{d}$ yield ratio [20]. This ratio has been studied experimentally as function of $\sqrt{s_{\mathrm{NN}}}$ [ [21] and a pronounced 'horn' structure arises at relatively low reaction energies, see Fig.2 Moreover there seems to be a raise in this ratio after a dip at intermediate energies.

This effect is due to a rather sudden modification of chemical conditions in the dense matter fireball: the rapid rise in strangeness $\bar{s}$ production below, and a rise in the anti-quark $\bar{d}$ yield above the 'horn'. The measured $\mathrm{K}^{+} / \pi^{+}$ratios are fitted 

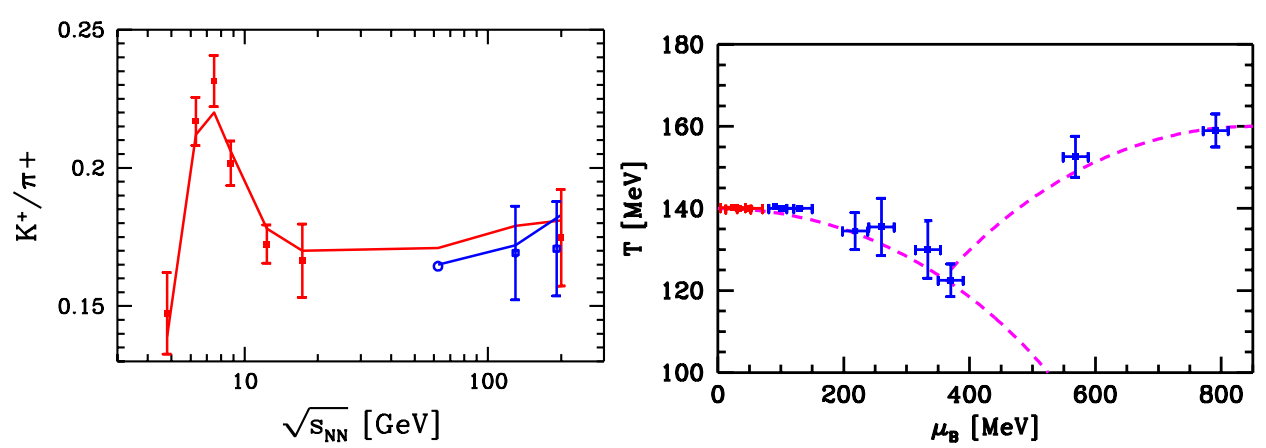

Fig. 2. On left $K^{+} / \pi^{+}$total yield ratio as function of $\sqrt{s_{\mathrm{NN}}}$ and its statistiacal hadronization model fit employing chemical non-equilibrium. At RHIC, the central rapidity yield fit is also shown. On right, the fitted values of hadronization temperature and baryo-chemical potential are presented in the $T-\mu_{\mathrm{B}}$ plane. The highest $\mu_{\mathrm{B}}$ entries on right correspond to the lowest $\sqrt{s_{\mathrm{NN}}}$ values on left [ [7].

well as is shown by the continuous line in Fig. 2 when allowing for chemical nonequilibrium at hadronization. Both total yield ratios and the central rapidity ratios (for RHIC) have been studied as shown on left in Fig.2

A fit to this horn generates an 'inverted horn' in the $T-\mu_{\mathrm{B}}$ plane, see right hand side of Fig. 2. The RHIC $d N / d y$ results are to outer left. They are followed by RHIC and SPS $N_{4 \pi}$ results. The dip corresponds to the 30 and $40 A \mathrm{GeV}$ SPS results. The top right is the lowest $20 \mathrm{AGeV}$ SPS and top $11.6 \mathrm{AGeV}$ AGS energy range. To guide the eye, we have added two lines connecting the fit results. We see that the chemical freeze-out temperature $T$ rises for the two lowest reaction energies 11.6 and $20 \mathrm{~A} \mathrm{GeV}$ to near the Hagedorn temperature, $T=160 \mathrm{MeV}$, of boiling hadron matter.

Such a non-trivial hadronization boundary, in the $T-\mu_{\mathrm{B}}$ plane, is the result of a complex interplay between the dynamics of heavy ion reaction, and the properties of both phases of matter, the inside of the fireball, and the hadron phase we observe. The dynamical effect, capable to shift the location in temperature of the expected phase boundary is due to the expansion dynamics of the fireball, see section 3 and effects of chemical non-equilibrium, see section 2 for full discussion. However, the behavior seen at lower reaction energies maybe in part due to presence of effectively massive quarks, which do not chemically equilibrate.

\section{Highlights}

Our objective has been to show that there is a good reason to expect that the behavior of the QGP formed in heavy ion collisions can deviate in a significant manner from expectations formed in study of equilibrium thermal QCD matter. We have described two main effects, the chemical non-equilibrium of strange quarks, and the pressure of flowing color charge acting on the vacuum, which, in our opinion, are at current level of knowledge relevant to the issues considered. Of most theoretical 
relevance and interest are the implications of non-equilibrium hadronization on the possible change in the location and nature of the phase boundary.

We have further argued that strangeness, and entropy, are well developed tools allowing the detailed study of hot QGP phase. We have shown that it is possible to describe the 'horn' in the $K^{+} / \pi^{+}$hadron ratio within the chemical non-equilibrium

statistical hadronization model. The appearance of this structure is related to a rapid change in the properties of the hadronizing matter.

\section{Acknowledgments}

Work supported by a grant from: the U.S. Department of Energy DE-FG02-04ER4131. LPTHE, Univ. Paris 6 et 7 is: Unité mixte de Recherche du CNRS, UMR7589.

\section{References}

1. A. Peikert, F. Karsch, E. Laermann and B. Sturm, Nucl. Phys. Proc. Suppl. 73, 468 (1999).

2. Z. Fodor and S. D. Katz, JHEP 0404, 050 (2004).

3. F. Karsch and E. Laermann, In R.C. Hwa, et al.: Quark gluon plasma III (2004) pp 1-59 (World Scientific, Singapore).

4. C. Bernard et al. [MILC Collaboration], Phys. Rev. D 71, 034504 (2005).

5. J. Rafelski and J. Letessier, arXiv:hep-ph/0506140, Euro. J. Phys. C in press.

6. J. Letessier, J. Rafelski and A. Tounsi, Phys. Rev. C 50, 406 (1994).

7. J. Letessier and J. Rafelski, "Hadron production and phase changes in relativistic heavy ion collisions," arXiv:nucl-th/0504028.

8. J. Letessier and J. Rafelski, Hadrons and quark-gluon plasma Cambridge Monogr. Part. Phys. Nucl. Phys. Cosmol. 18 1-397 (2002). Available on line at http://site.ebrary.com/pub/cambridgepress/Doc?isbn=0521385369

9. J. Rafelski and J. Letessier, Phys. Lett. B 469, 12 (1999).

10. T. S. Biro et al.,Phys. Rev. C 48, 1275 (1993).

11. D. Pal, A. Sen, M. G. Mustafa and D. K. Srivastava, Phys. Rev. C 65, 034901 (2002).

12. Z. J. He et al., Phys. Rev. C 69, 034906 (2004).

13. J. Letessier, A. Tounsi and J. Rafelski, Phys. Lett. B 389 (1996) 586.

14. J. Letessier and J. Rafelski, Phys. Rev. C 67, 031902 (2003).

15. J. Rafelski and J. Letessier, Phys. Rev. Lett. 85, 4695 (2000).

16. T. Csorgo and J. Zimanyi, Heavy Ion Phys. 17, 281 (2003).

17. J. Letessier et al., Phys. Rev. Lett. 70, 3530 (1993) arXiv:hep-ph/9711349.

18. J. Rafelski and B. Muller, Phys. Rev. Lett 48, 1066 (1982); 56, 2334E (1986).

19. J. Rafelski, J. Letessier and G. Torrieri, Phys. Rev. C 72, 024905 (2005).

20. N. K. Glendenning and J. Rafelski, Phys. Rev. C 31, 823 (1985).

21. M. Gazdzicki et al. [NA49 Collaboration], J. Phys. G 30, S701 (2004). 